\title{
On trapped modes in the LHC recombination chambers: numerical and experimental results.
}

\author{
D. Brandt, F. Caspers, F. Ruggiero, L. Vos, \\ CERN, Switzerland. \\ A. Mostacci, M. Migliorati, L. Palumbo, \\ University of Rome "La Sapienza" and LNF-INFN, Italy. \\ B. Spataro, \\ LNF-INFN, Italy. \\ Derun Li, \\ Lawrence Berkeley National Laboratory Berkeley, U.S.A.
}

\begin{abstract}
The recombination chamber in LHC (Large Hadron Collider) allows the separated proton beams to merge into a common vacuum chamber surrounding the interaction points. It has been subject of through studies concerning its interaction with the circulating beam. In this paper we present the numerical and experimental results of our investigation. We show that in the smooth transitions between pipes of different diameters a trapped mode may exist. The mode results to be not harmful for the LHC operation, both for the beam stability and power loss.
\end{abstract}

Administrative Secretariat

LHC Division

CERN

CH-1211 Geneva 23

Switzerland

Geneva, August 19, 2002 
The recombination chamber is the piece of equipment which allows the separated beams to merge into the common vacuum chamber surrounding the interaction points. Given its particular shape (see Fig. 1), it is often referred to as the Y-chamber. Before arriving at the interaction point, each beam will travel from a small beam pipe, the standard LHC vacuum chamber, through a pipe gradually increasing in diameter to finally reach the larger chamber around the interaction point, namely the transition is tapered. The intersection of the two conical tapers generates a metallic sheet with an edge of parabolic shape which makes even smoother the transition. The design and the production of these chambers is under the responsibility of the Lawrence Berkeley National Laboratory (LBNL) in the USA.

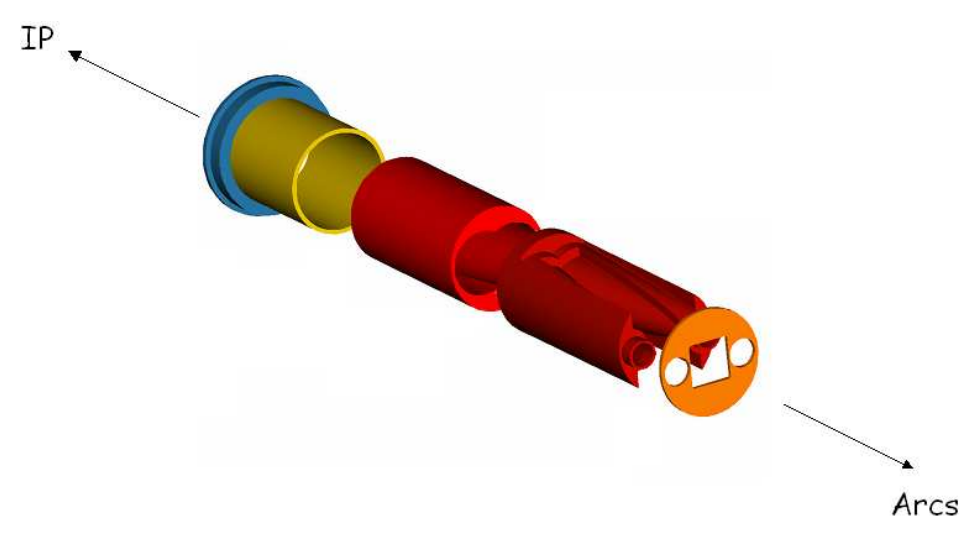

Figure 1: The beam tube transition where two small beam tubes (coming from the arcs) merge to a single large one at the Interaction Point (IP).

The design of these smooth transitions between two pipes of different diameters requires some careful thoughts as far as the interactions of the electromagnetic fields with the structure are concerned. The coupling impedance of such transition has been investigated numerically for both the real Y-chamber geometry [1] (see Fig. 2) and for a simplified rectangular geometry [2] (see Fig. 3). The rectangular geometry reproduces well the actual (circular) Y-chamber geometry, only when a V-shaped metal sheet is placed in front of the taper (see Fig. 3). The main conclusions have been the discovery of a trapped mode due to the taper. Such a metal sheet strongly reduces the $R / Q$ of that mode, but does not eliminate it completely.

The relevant geometries of the device and models are described in Sec. 2. The results of the numerical simulations are presented in Sec. 3 and the bench measurements performed to asses the presence of the trapped mode are discussed in Sec. 4.

\section{The recombination chamber}

The recombination chamber is located on each side of the experimental areas. The chamber is composed of a common cylinder of diameter $D=180 \mathrm{~mm}$ which then splits into two smaller cylinders of $d=54 \mathrm{~mm}$ diameter each. For the sake of the present investigation, the cylindrical geometry has been first replaced by a rectangular one, such that the diameter of the real pipe is the same as the size of the square retained (i.e. the real structure can be inscribed in the simulated one). The study of the rectangular geometry has been performed for two main reasons: the simpler geometry allows us to 

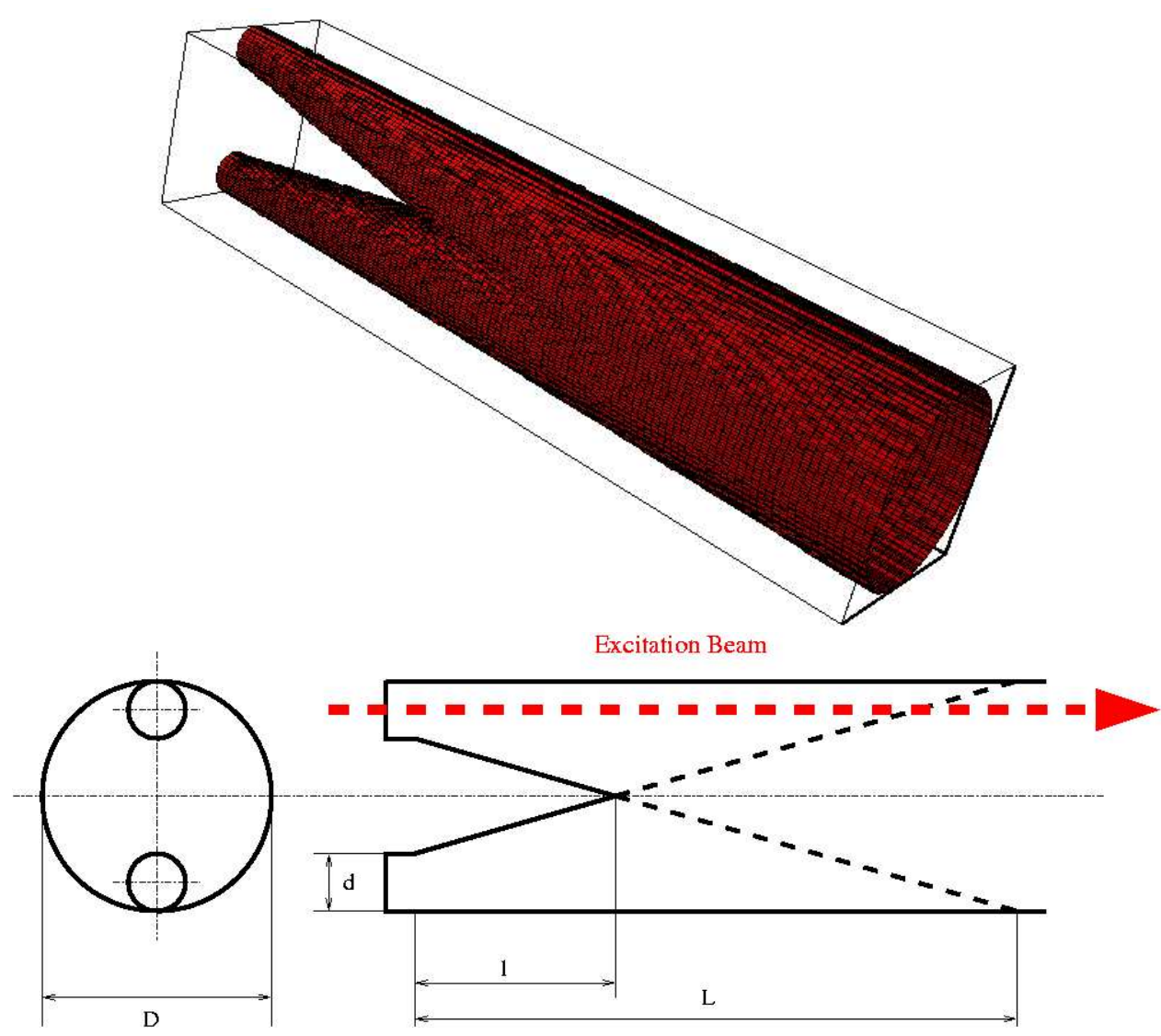

Figure 2: Relevant geometry for the Y-junction. The upper picture shows the model used in the MAFIA simulations (circular geometry). The cross sections are shown in the lower picture $(L=852 \mathrm{~mm}, l=260 \mathrm{~mm}, d=54 \mathrm{~mm}, D=180 \mathrm{~mm})$ where the arrow indicates the path of the exciting beam.

separate the effects of the taper from that of the metal sheet and for such a geometry it is easier to make a scaled prototype for bench test measurements.

The real structure and the simplified one used for the simulations with MAFIA [3] are illustrated in Fig.s 2-3. At the junction of the two small chambers, the inner structure is continued by a kind of metal sheet. It will be shown later that the shape and the length of this metal sheet play an important role as far as the interaction with electromagnetic fields is concerned.

\section{$3 \quad$ Numerical simulations}

\subsection{Rectangular geometry}

In spite of the simplified rectangular geometry, the structure remains difficult to handle with MAFIA and the simulation runs are very time consuming. In order to optimise both the available resources and the accuracy of the results, the following additional simplifications have been considered:

- The Y-chamber on the left of the interaction point (entry in the straight section) is treated independently from that on the right of the interaction point (exit from the straight section), although, from an impedance point of view, the two Y-chambers represent a common single structure.

- In the real structure, the trajectory of the beam is slightly inclined when going 


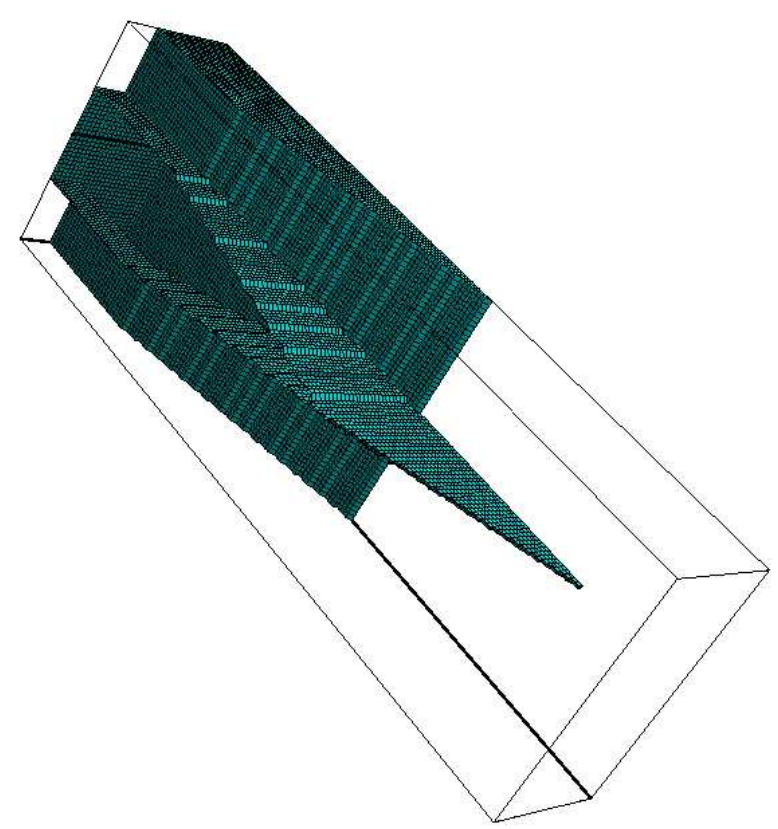

Figure 3: Rectangular model of the Y-chamber used for MAFIA simulations (half structure because of symmetry).

from the arc vacuum chamber into the common chamber of the straight section. This angle is very small and has been neglected in the simulations. Furthermore, it has been assumed that the beam travels in the centre of the smaller pipe radius chamber.

- Because of the above approximations, the geometry of the structure is $y z$-symmetric, and it is thus possible to simulate only half of the structure (see Fig. 3).

- In order to get a detailed understanding of the effect of the metal sheet, the length of the latter has been varied from zero (no metal sheet) up to the nominal length.

For the present investigations, we have conducted 3D MAFIA simulations in both frequency and time domain. In order to get some insight into the electromagnetic interaction between the beam and the Y-chamber, and therefore to obtain the coupling impedance of this device, we first compute the wake potential of a Gaussian bunch (r.m.s. bunch length of $1.5 \mathrm{~cm}$ ) over a distance of $6 \mathrm{~m}$ behind the bunch. A subsequent Fourier transform of the wake potential yields the real and the imaginary part of the impedance for the Y-chamber.

\subsubsection{Y-chamber without metal sheet}

The impedance is illustrated in Fig. 4 (real part) and in Fig. 5 (imaginary part). Although the cut-off frequency of the common vacuum chamber is estimated to be around $850 \mathrm{MHz}$ (TE only), one observes the presence of a strong trapped mode at $1.026 \mathrm{GHz}$. Since the frequency of this mode is higher than the cut-off frequency of the common chamber, it could have been expected that the mode would propagate. As matter of facts, this mode is trapped around the sharp edge of the tapers and is weakly coupled to the modes of the wider chamber. At frequencies lower than the first peak, the real part of the coupling impedance is greater than zero since we are simulating a single transition from a narrow pipe to a wider region [4].

A more detailed investigation resulted in the following observations: 


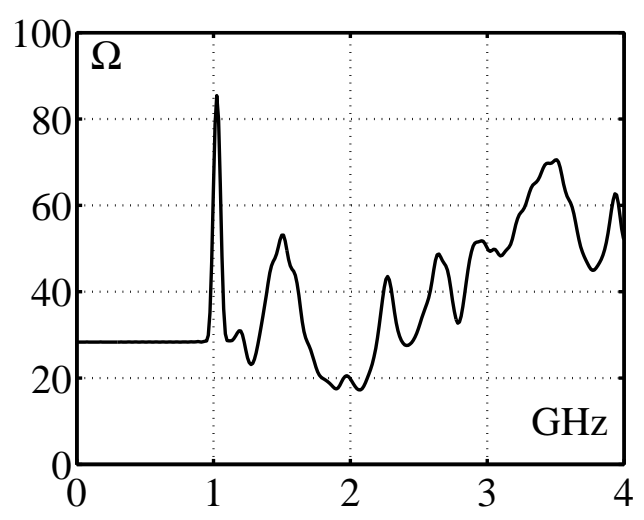

Figure 4: Real part of the longitudinal coupling impedance for the Y-chamber (no metal sheet, rectangular shape). The sharp resonance is at $1.026 \mathrm{GHz}$.

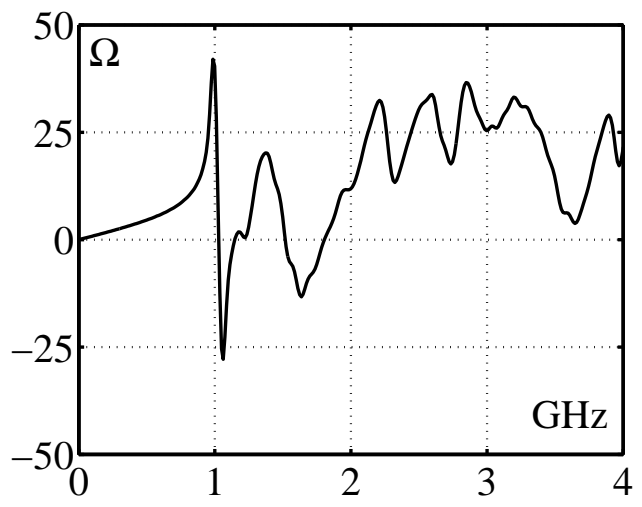

Figure 5: Imaginary part of the longitudinal coupling impedance for the Y-chamber (no metal sheet, rectangular shape).

- This resonance is introduced by the taper joining the small vacuum chamber of the arc to the larger chamber of the common section.

- The longitudinal impedance of the structure has been also computed in the frequency domain (magnetic boundary conditions at both ends of the structure). It results in a shunt impedance ${ }^{1)}$ of $54 \mathrm{k} \Omega$ at the trapped mode frequency and a quality factor $Q$ of 25000 . Since the revolution frequency of the LHC $(11 \mathrm{kHz})$ is smaller than the width of the trapped mode $(41 \mathrm{kHz})$, coupled bunch motion would be likely driven [5].

\subsubsection{Y-chamber with metal sheet}

The beneficial effect of the metal sheet is to ensure an even smoother transition. As shown below, it strongly reduces the dangerous effects observed above; however, it does not allow for their complete cancellation.

In order to better understand the role of the metal sheet, calculations have been performed for different longitudinal lengths. The main qualitative feature is that increasing the length has the tendency to damp the dangerous resonant mode. The results for the impedance of the rectangular model of the structure are presented in Fig. 6 (real part).

1) The shunt impedance $R_{s h}$ is defined analogously to electric circuits, i.e. $P=V^{2} /\left(2 R_{s h}\right)$ where $P$ is the power lost in a given cavity when a voltage $V$ is applied. 
The metal sheet is $50 \mathrm{~cm}$ long, to simulate the LBNL design. As can be seen from the real part of the impedance, the trapped mode has been strongly damped. Admittedly, the mode is much weaker and is not able to excite coupled bunch modes. Moreover, in case the lines of the bunch spectrum would coincide with the frequencies of this mode, this would induce a power deposition of the beam into the Y-chamber of the order of 50 Watts, which is rather small.

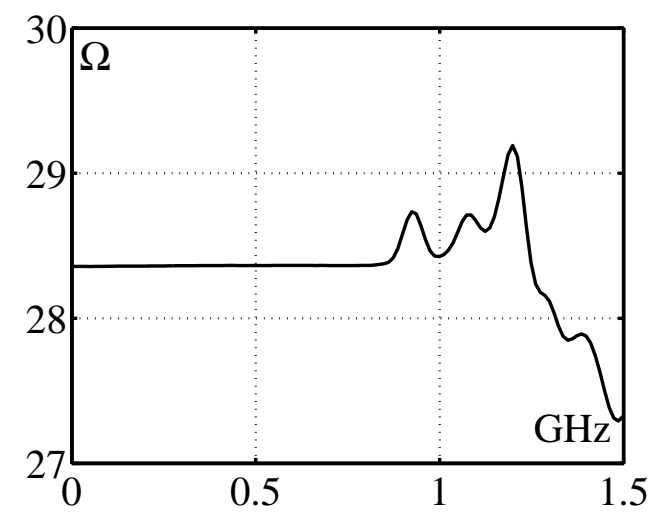

Figure 6: Real part of the longitudinal coupling impedance for the Y-chamber with $50 \mathrm{~cm}$ metal sheet (rectangular shape).

As far as the low frequency inductance of the Y-chamber is concerned, a complete structure (entry piece and exit piece) has been simulated. The corresponding imaginary part of the impedance is presented in Fig. 7 and yields a $Z / n \approx j 0.1 \mathrm{~m} \Omega$, which can be considered as non-negligible, but acceptable [2].

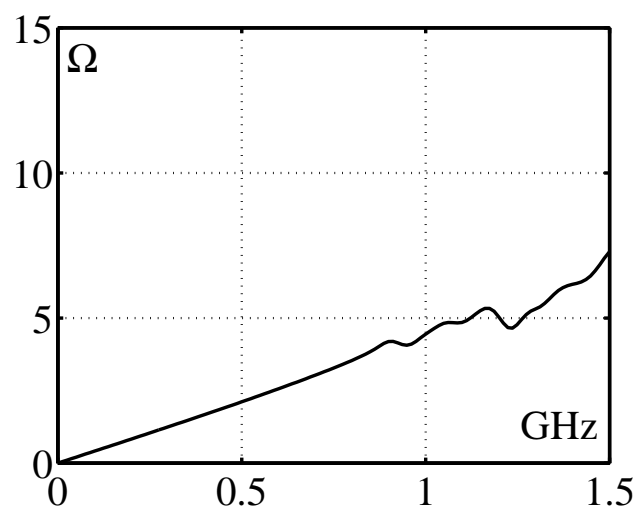

Figure 7: Imaginary part of the longitudinal coupling impedance for the Y-chamber with metal sheet (rectangular shape, global structure).

\subsection{Real geometry}

The results obtained with the rectangular geometry have been confirmed by the simulations performed for the real geometry (Fig. 2) for which the wake potential of a Gaussian bunch (r.m.s. bunch length of $1.2 \mathrm{~cm}$ ) has been calculated over a distance of $3 \mathrm{~m}$ behind the bunch. A subsequent Fourier transform of the wake potential yields the real and the imaginary part of the impedance. The real and imaginary part of the impedance are shown in Fig. 8. 


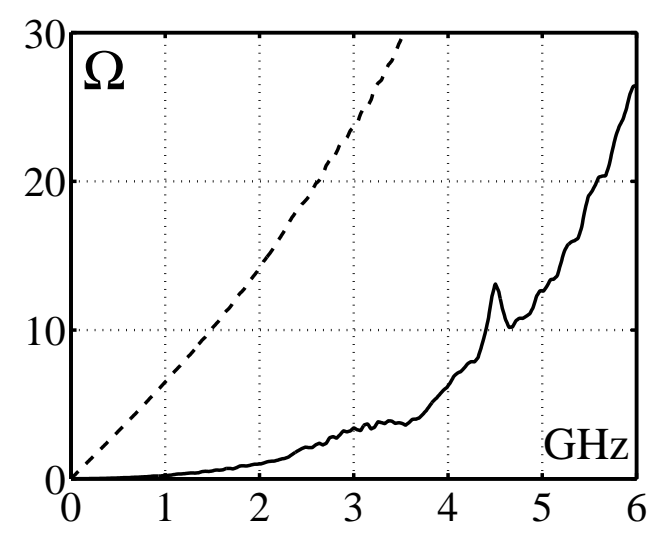

Figure 8: Real part (solid line) and imaginary part (dashed line) of the impedance for the Y-chamber (actual structure, circular shape).

The existence of a weak trapped resonance around at $4.5 \mathrm{GHz}$ is clearly shown; the cut-off frequencies for TM modes of the combined chamber are $1.06 \mathrm{GHz}$ and 4.42 $\mathrm{GHz}$ for the smaller tubes. It also confirmed that the smoother transition introduced by the metal sheet with parabolic edge, allows the mode to propagate in the wider common chamber. The residual mode should not be a concern to the beam dynamics; in fact it is characterised by a small $R / Q$ value, its frequency is high compared to the LHC bunch spectrum (nominal r.m.s bunch length of $7.5 \mathrm{~cm}$ ) and therefore the excitation of this mode is weak. The broad band impedance [5] of this mode is also very small, namely $Z / n$ $\approx j 75 \mu \Omega$.

\section{$4 \quad$ Experimental investigation}

For practical reasons, we consider a rectangular scaled model of the LHC recombination chamber. Figure 9 shows half of the whole structure and the $y z$-plane is the symmetry plane. From the squared common region (of side $a=66 \mathrm{~mm}$ ), two (squared) beam pipes of side $b=18 \mathrm{~mm}$ take off; the transition taper is long $c=85 \mathrm{~mm}$. The structure of Fig. 9 is placed inside a box of squared cross-section of side $a$.

A wire is located on the beam trajectory (light blue line) and it results in a coaxial transmission line whose properties are related to the behaviour of a particle going through the tapered transition. In particular the energy lost by a relativistic particle travelling in the structure can be estimated from the transmission attenuation between the two ports connected by the wire. Therefore any trapped mode possibly excited by the beam in the transition of Fig. 9 can be excited with the wire.

\subsection{HFSS Simulations of the scaled prototype}

To check the feasibility of the measurements, a campaign of HFSS [6] simulations has been performed about the actual bench set-up (Fig. 9) with a "perfectly conducting" wire of square cross section $(2 \mathrm{~mm} \times 2 \mathrm{~mm})$. The taper alone (no metal sheet) has been studied first since a strong trapped mode was expected. A typical result is shown in Fig. 10 where the significant notch in the $S_{12}$ at $2.737 \mathrm{GHz}$ is a clear sign of the presence of the mode. The simulated structure has no loss mechanism since the structure is perfectly conducting, and the minimum $S_{12}$ is approaching a value close to zero $(-\infty$ in a db scale). In the real prototype (brass and aluminium, see Sec. 4.2) the resistive losses wide the resonance, but they do not eliminate it. 


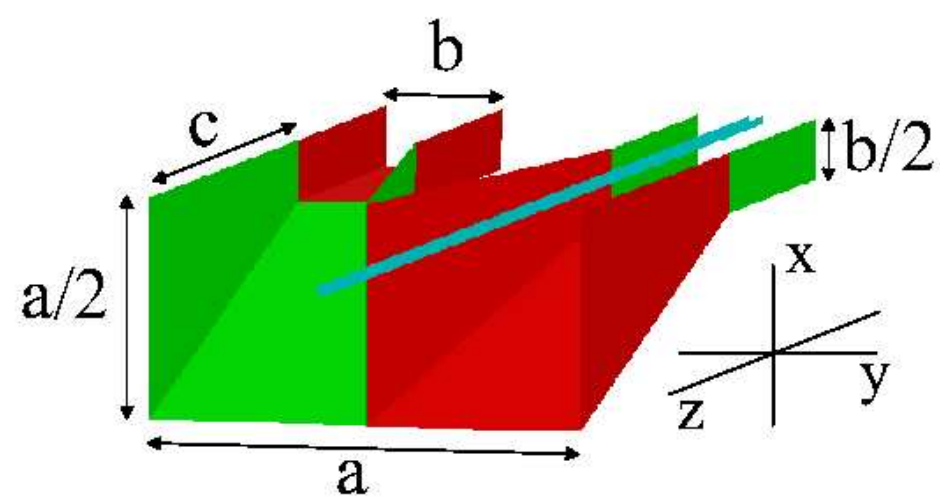

Figure 9: Bench measurement geometry. The small tubes represent the two beam pipes merging into the common region through the tapers. The light blue line represents the wire used to simulate the behaviour of the beam both in the bench measurement and in the HFSS simulations (see sec. 4.1). Only the lower half structure is shown and there is no metal sheet $(a=66 \mathrm{~mm}, b=18 \mathrm{~mm}, c=85 \mathrm{~mm})$.

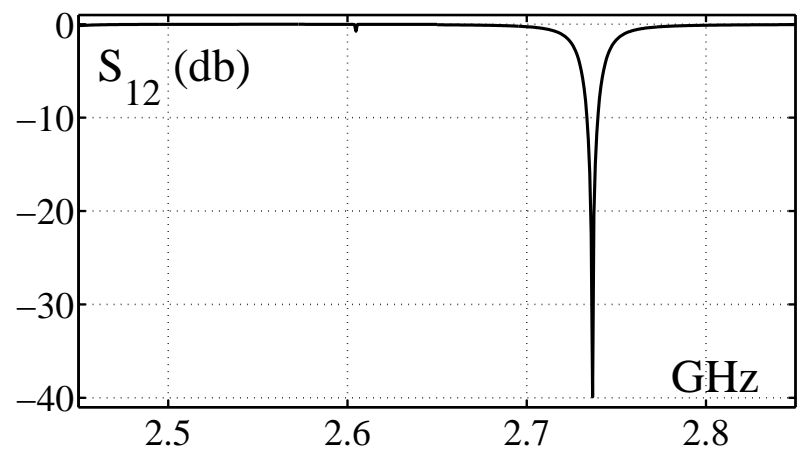

Figure 10: Transmission coefficient at various frequencies from HFSS simulation. The structure is ideal, i.e. no losses and perfect matching; thus the $S_{12}$ is unitary (0 db) except close to the resonance where the trapped mode is $(2.737 \mathrm{GHz})$.

It is interesting to look into the field configuration inside the structure. When the transmission is minimum, the power entering the structure from the input port (lower part of Fig. 11) is reflected at the taper and the field has a typical standing wave pattern as in Fig. 11a which shows the maximum amplitude of the electric field. The field is concentrated around the wire, as expected for the fundamental mode in any coaxial structure. There is also a strong field concentration close to the wedge of the taper which confirms the existence of the trapped mode; on the contrary, a very small amount of field reaches the end of the two small waveguides indicating big losses for the beam at that frequency. The field pattern is completely different at $2.5 \mathrm{GHz}$, i.e. in the middle of the transmission plateau of Fig. 10. The field is guided by the coaxial wire and there is a full transmission of power between the two ports connected by the wire. The electric field amplitude is maximum along the wire (Fig. 11b) and it is inversely proportional to the distance from the wire. A beam travelling along the wire direction will not excite any trapped mode at this frequency.

In conclusion, the trapped mode is present also in the numerical analysis of our bench set-up and it can be measured by looking for a strong notch in the transmission 


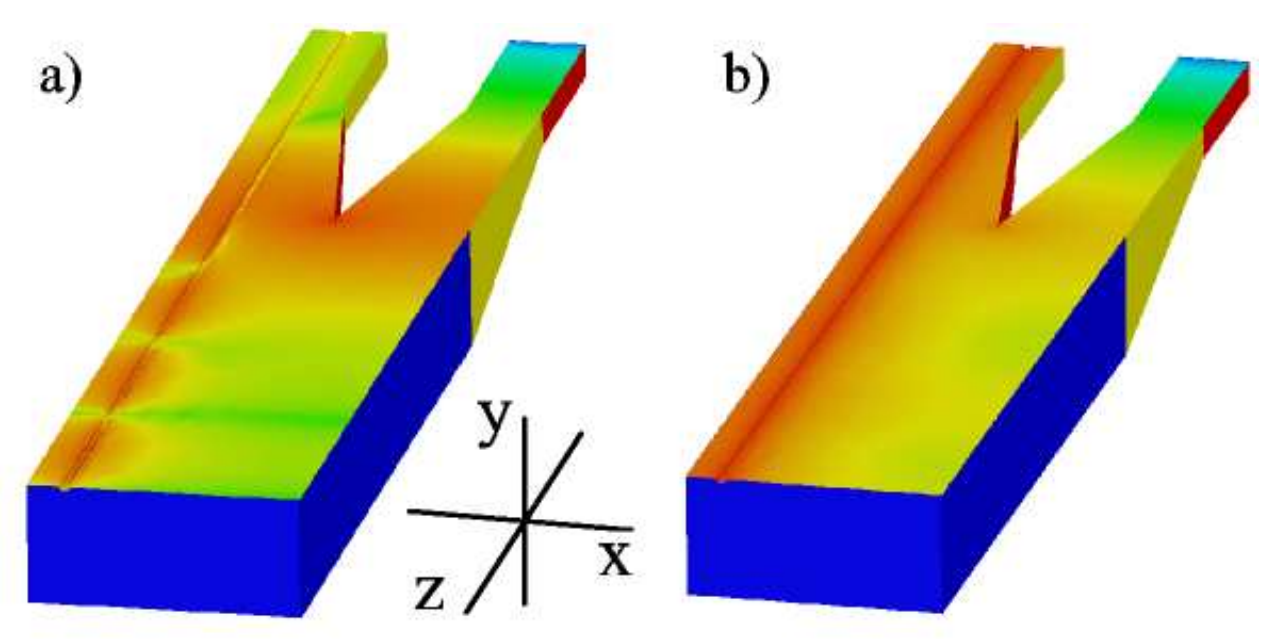

Figure 11: Amplitude of the electric field (maximum value). The left plot is the field at $2.737 \mathrm{GHz}$ where the transmission notch is; the bigger part of the e.m. power is in the trapped mode at the wedge. On the contrary in the right plot $(2.5 \mathrm{GHz})$ the field is propagating and it remains close to the wire. The (logarithmic) colour scale is such that the maximum amplitude is red and the minimum one is blue (scaling as the rainbow colours). The input port is at the bottom in both the pictures.

coefficient of a coaxial wire structure.

\subsection{Experimental set-up}

The prototype built is relatively simple and cheap; its dimensions have been chosen to be a smaller model of the recombination chamber and the relative proportions among the different parts have been maintained as in the Y-chamber design done by LBNL. The common region is modelled by an aluminium box with square cross-section $(33 \mathrm{~mm} \times 33 \mathrm{~mm} \times 1 \mathrm{~m})$ and the tapered region is obtained with a brass transition, as shown in Fig. 12. A copper wire of $0.4 \mathrm{~mm}$ of diameter is stretched between the two connectors in the lower part of the brass taper; matching resistors are also included and their values are chosen according to Appendix A.

An important issue for getting reproducible results, is to assure good contacts; the contact between the brass transition and the external box is improved by using proper RF-fingers (the RF contacts of Fig. 12a). The two halves of the structure are also pressed one against the other by external screws.

Two different measurements have been performed and will be discussed in details, namely the taper alone, and with a metal sheets obtained from a $0.02 \mathrm{~mm}$ thick copper foil.

\subsection{Experimental results}

A first set of measurements has been performed on the tapered transition alone, i.e. without the metal sheet. The transmission coefficient $S_{12}$ is reported in Fig. 13. A strong notch at $2.753 \mathrm{GHz}$ indicates the trapped mode; such a frequency is close to the numerical predictions, that is $2.737 \mathrm{GHz}$ from HFSS. The fluctuations on the flat part of the $S_{12}$ may depend on the non perfect matching. The growing of $S_{12}$ around $3 \mathrm{GHz}$ (Figs. 13-14) is due to fact that the matching is realised with simple carbon resistors and at that frequency the parasite capacitance and inductance start playing their role and the 

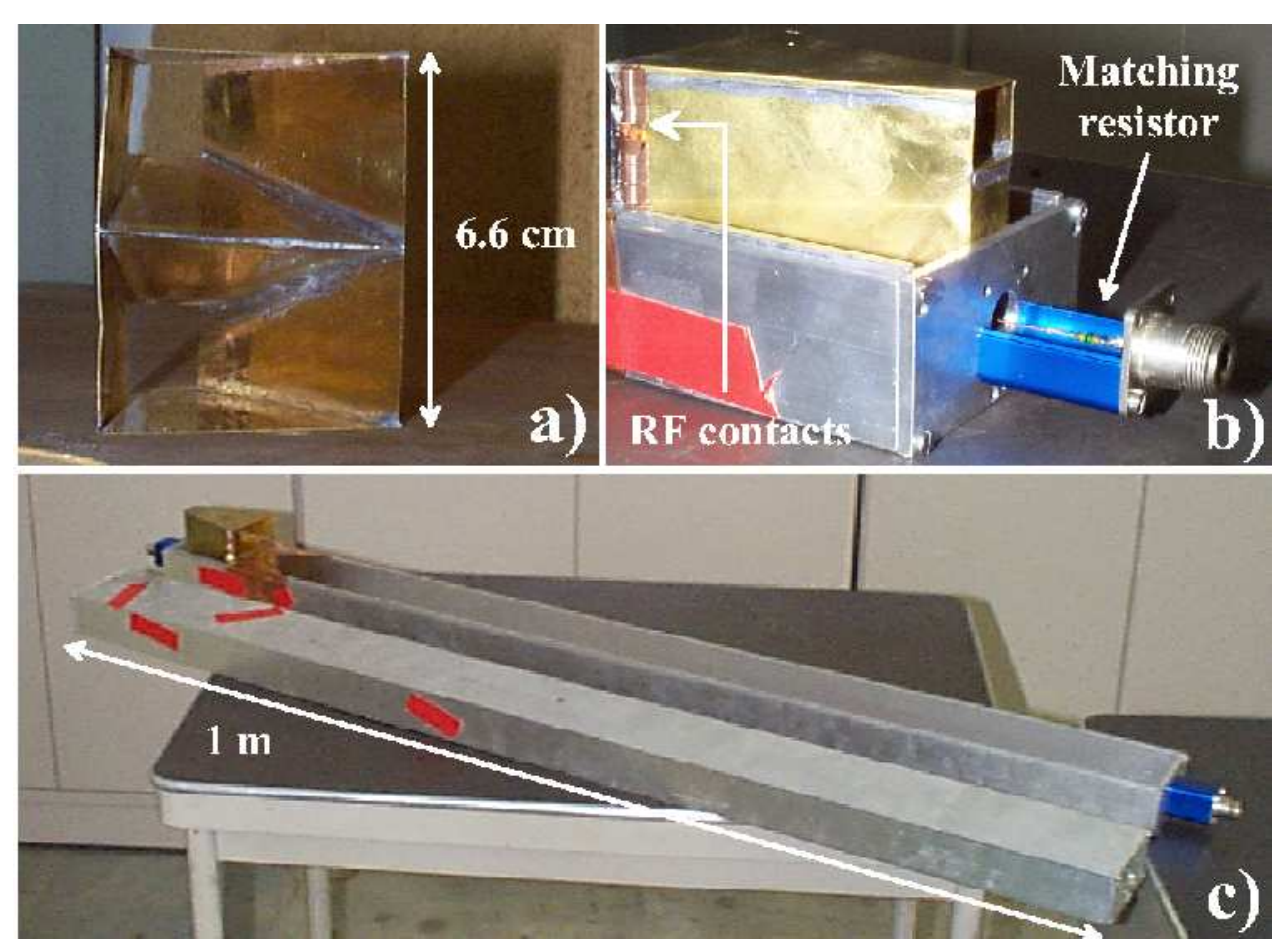

Figure 12: Bench measurement prototype. The taper is made of brass and proper RF contacts are soldered on it. A matching resistor joins each $\mathrm{N}$-connector to the copper wire (0.4 mm of diameter). The tapered transition is inserted in aluminium box of one meter length. A "short" V-shaped metal sheet is soldered to the taper.

matching condition is not fulfilled anymore.

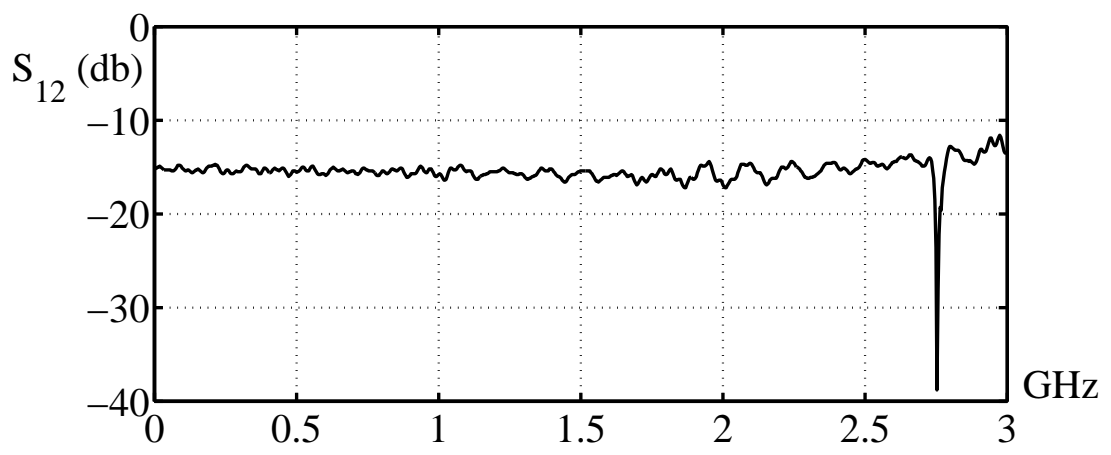

Figure 13: Transmission coefficient versus frequency for the tapered structure (no metal sheet). The strong resonant decrease in the transmission clearly indicates the presence of a trapped mode at $2.753 \mathrm{GHz}$.

Including a V-shaped metal sheet in the bench prototype, the trapped mode should reduce its amplitude and shift down in frequency. In Fig. 14 results are given for the case of a $20 \mathrm{~cm}$ long, V-shaped metal sheet soldered on the taper. This length has be chosen to reproduced the influence of the parabolic metal sheet present in the actual design of the LHC transition region. The measurements show a complete suppression of the trapped mode, while the simulations predicted a residual effect. Our measurement sensitivity was not high enough to measure such small effects in an unambiguous manner. Introducing 
some perturbation (metal object) close to the taper, we observed clearly some effect, meaning that there is still some electromagnetic energy trapped in the vicinity of the taper; anyway there is not a clear sign of this in the $S_{12}$ of the whole structure.

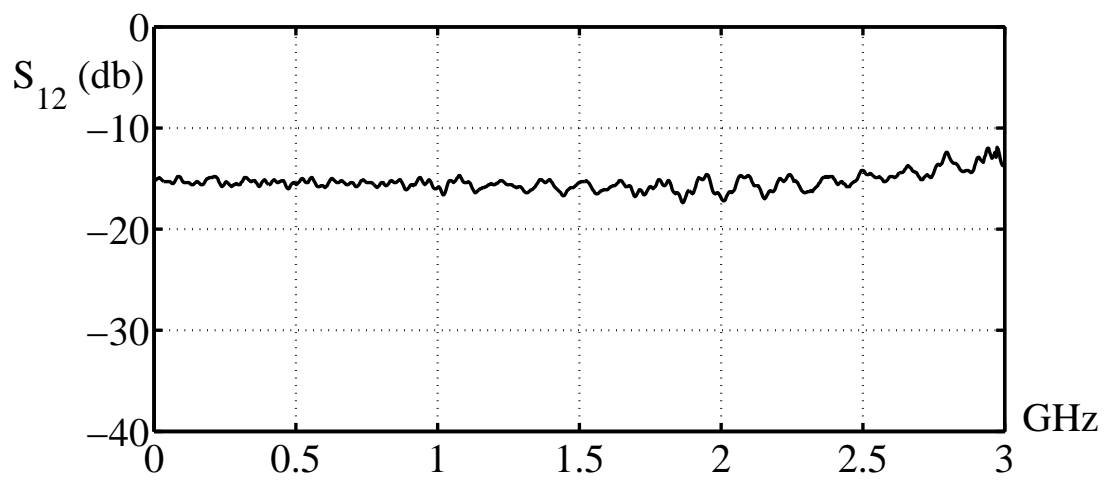

Figure 14: Transmission coefficient versus frequency for the tapered structure with a Vshaped metal sheet $20 \mathrm{~cm}$ long. The trapped mode related notch is so much reduced that it can not be seen in the measurements.

\section{Conclusion}

In this paper we present the relevant results of a numerical and experimental study of the recombination chamber of LHC. A trapped mode is predicted by the simulations. Fortunately such a mode is strongly reduced by the metal sheet present in the actual design, as confirmed by MAFIA simulations [2]. We have designed and built a simple bench model which resembles the Y-chamber, but it is smaller (about 3 times) and it has rectangular cross-sections (instead of circular ones). The experimental results agree pretty well with numerical simulations and they both confirm the existence of the trapped mode and the beneficial effect of the metal sheet which substantially reduces the influence of the mode.

\section{$6 \quad$ Acknowledgements}

The authors are grateful to B. Zotter for useful comments and discussions. J. MDarve substantially helped in finding a simple (and cheap) way to build the model of the taper.

\section{References}

[1] G. Lambertson, D. Li, W.C. Turner, Broadband impedance calculations of the TAN Vacuum Chamber for the Large Hadron Collider (LHC), Proceedings of the 2001 Particle Accelerator Conference (PAC01), Chicago, June 2001.

[2] B. Spataro et al., Impedance of the LHC recombination chambers, LHC Project Note 254, May 2001.

[3] R. Klatt et al., MAFIA - a three dimensional electromagnetic CAD system for magnets, RF structures and transient wake-field calculations, Proceedings of the 1986 Linear Accelerator Conference, Stanford Linear Accelerator Centre, SLAC-Report 303, June 1986 (pp. 276-278).

[4] L. Palumbo, V.G. Vaccaro and M. Zobov, Wake fields and impedance, CAS Cern Accelerator School, Advanced school on accelerator physic, Rhodes 1994, LNF-94/041 (1994). 
[5] A. W. Chao, Physics of Collective Beam Instabilities in High Energy Accelerators, Wiley-Interscience, New York, 1993.

[6] See World Wide Web address, www. ansoft.com. 


\section{A Resistive matching networks for the bench set-up}

As discussed above, our bench set-up is a coaxial line obtained by inserting a copper wire in our Device Under Test (DUT). The transition between such a coaxial line and the cables connecting the Vector Network Analyser (VNA) is not automatically matched. Let us assume that the coaxial line has a characteristic impedance of $Z_{c}$ and that the VNA is calibrated, i.e. the cables have the same impedance $Z_{0}=50 \Omega\left(<Z_{c}\right)$. The measurement set-up schematically looks like Fig. 15, where the transition between the different transmission lines is done with matching networks to minimise (ideally to avoid) reflections. In the ideal case, a wave going from the coaxial line of the DUT into the cables (see dashed arrows in Fig. 15) is not reflected back and this situation is called "internal matching". Similarly a wave travelling from the cables to the coaxial line (solid arrows in Fig. 15) is not reflected either and we will refer to it as "external matching".

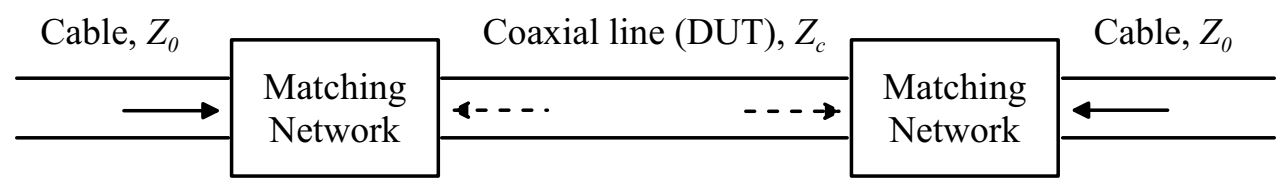

Figure 15: Schematic view of the bench measurement set-up. The Device Under Test (DUT) is the line in the middle with characteristic impedance $Z_{c}$. External cables, with impedance $Z_{0}=50 \Omega$ connect the structure to the VNA and their terminations are assumed to be perfectly matched. The solid arrows represent waves going from the $Z_{0}$ transmission line to the coaxial line $\left(Z_{c}\right)$ while the dashed lines represent waves in the opposite directions. An ideal matching network should avoid reflections for all those waves.

Different types of matching networks are possible, but we will focus only on resistive ones. They are easy to build and they work well at low frequencies; at high frequencies, the matching depends on the quality of the resistors (parasite capacitance and inductance). For narrow band (or high frequency) measurements, better matching can be achieved by conical transitions (which have to be designed ad hoc).

To achieve both "internal" and "external" matching, a network of at least two resistors is needed. Anyway to prevent only multiple reflections at the ends of the coaxial line without caring of the reflected signal at the transition from the cable to the coaxial line, only one resistor is enough. The single resistor matching network has also the advantage of having a wider bandwidth than the networks with multiple resistors. In other words, the condition for "internal matching" only can be satisfied with a single resistor such that

$$
Z_{c}=Z_{0}+Z_{\text {match }}
$$

where the matching resistance $Z_{\text {match }}$ is connected as in Fig. 16. Equation (1) simply states that the impedance seen from the coaxial line looking towards the cable is equal to impedance of the incident wave.

To measure $Z_{c}$ we used a kind of "Time Domain Reflectometry", that is observing the signal reflected by the unmatched transmission line with a synthesised step excitation (called "low frequency step" in the VNA jargon). The calibrated $S_{22}$ for the (unmatched) coaxial line is shown in Fig. 17a, where the different "stairs" are the multiple reflections at the opposite end of the structure (HP8753D with time domain option). From the amplitude $\Delta \Gamma$ of the reflection coefficient one gets the impedance of the line

$$
Z_{c}=Z_{0} \frac{1+\Delta \Gamma}{1-\Delta \Gamma}=Z_{0} \frac{1+0.714}{1-0.714}=299 \Omega
$$




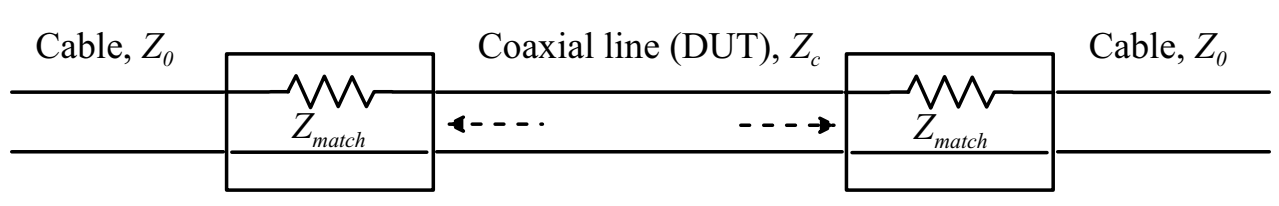

Figure 16: One resistor matching network. Only matching in one direction can be achieved. The resistor is chosen to avoid reflections only for waves going from the central coaxial line $\left(Z_{c}\right)$ to the cables $\left(Z_{0}\right)$, that is "internal matching".

since $Z_{0}=50 \Omega$. According to Eq. (1), $Z_{\text {match }}=Z_{c}-Z_{0}=249 \Omega$; for practical reasons, then, the two matching resistors actually soldered between the wire and the connectors are of $240 \Omega$ and they are visible in Fig. 12b. The reflection coefficient of the matched line is shown in Fig. 17b, where the multiple reflections disappeared at the price of an increased reflection of the signal coming from the network (about $80 \%$ of the input signal is reflected versus only $65 \%$ without resistors on the wire).
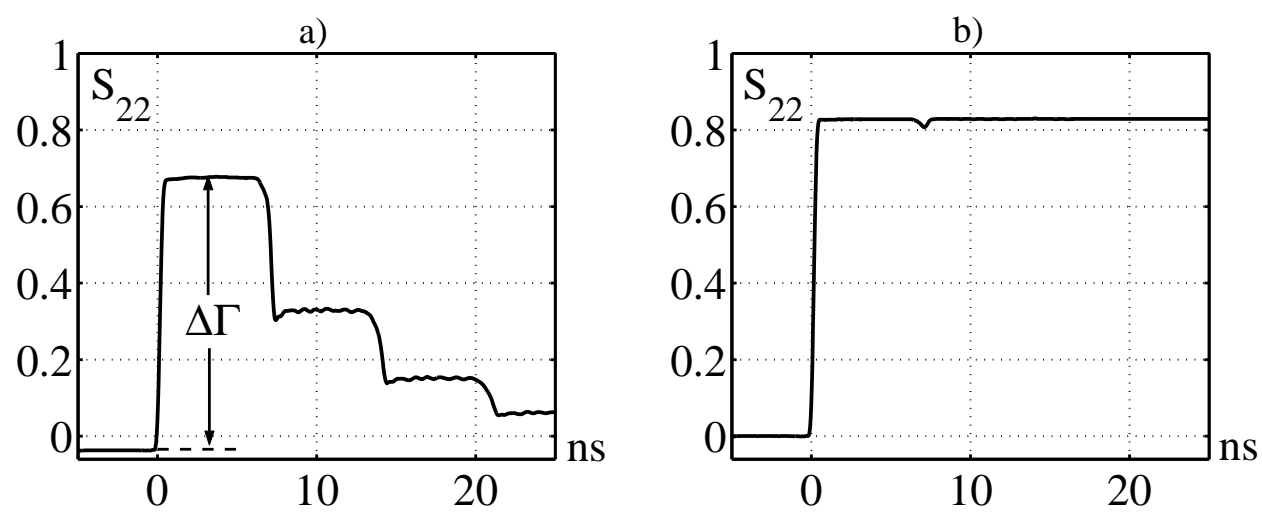

Figure 17: Reflection coefficient measurement in the time domain. The left plot is measured on the non-matched coaxial line while the right one is after including suitable matching resistors depending on the $\Delta \Gamma$ of the non-matched line $(\Delta \Gamma=0.714)$. 